\title{
Acknowledgment of Reviewers, 2008
}

The success of Phytopathology depends on the quality of manuscripts submitted by authors and on the care and competence with which they are reviewed. It is the policy of the Editorial Board to solicit reviews for manuscripts from specialists who are most qualified to review them. In addition to members of the Editorial Board, the individuals listed below provide constructive critical reviews of one or more manuscripts during the past year. Their names are published here in grateful appreciation of their contribution to the journal and to the science of phytopathology. doi:10.1094/PHYTO-99-1-0004

Accotto, G. P., Plant Virology Institute - CNR, Torino, ITALY Acosta-Leal, R., Texas A\&M University, Amarillo, TX, U.S.A. Adaskaveg, J. E., University of California, Riverside, CA, U.S.A. Aegerter, B., University of California, Stockton, CA, U.S.A. Agindotan, B. O., University of Illinois, Urbana-Champaign, Urbana, IL, U.S.A. Ajwa, H., University of California, Davis, Salinas, CA, U.S.A.

Akimitsu, K., Kagawa University, Miki, Kagawa, JAPAN Aldwinckle, H., Cornell University, Geneva, NY, U.S.A. Alma, A., Grugliasco, Torino, ITALY

Alvarez, A., University of Hawaii, Honolulu, HI, U.S.A

Anderson, J., USDA-ARS Purdue University, West Lafayette, IN, U.S.A.

Andrivon, D., INRA, Domaine de la Motte, Le Rheu, FRANCE

Angelini, R., University of Rome, ITALY

Armstrong, M. R., SCRI, Dundee, UNITED KINGDOM

Atallah, Z., University of California-Davis, Salinas, CA, U.S.A.

Avenot, H. F., University of California Davis, Parlier, CA, U.S.A.

Bach, E., Uninove, São Paulo, Santo André, BRAZIL

Bacon, C. W., USDA-ARS, Athens, GA, U.S.A.

Bai, G., USDA, Manhattan, KS, U.S.A

Bandla, M., USDA, Riverdale, MD, U.S.A.

Bar-Hen, A., CNRS, Paris cedex, FRANCE

Bar-Joseph, M., ARO, Bet Dagan, ISRAEL

Batuman, O., University of California, Davis, CA, U.S.A.

Beaulieu, C., Université de Sherbrooke, Sherbrooke, QC, CANADA

Bejarano, E. R., Universidad de Malaga, Malaga, Spain

Bennett, R. S., USDA-ARS, Shafter, CA, U.S.A.

Bent, A., University of Wisconsin, Madison, WI, U.S.A.

Berger, P., USDA-APHIS PPQ, Raleigh, NC, U.S.A.

Bergstrom, G. C., Cornell University, Ithaca, NY, U.S.A.

Bisaro, D. M., Ohio State University, Columbus, OH, U.S.A.

Block, C. C., USDA, Iowa State, Ames, IA, U.S.A.

Boag, B., SCRI, Dundee, UNITED KINGDOM

Bolton, M. D., U.S. Department of Agriculture, St. Paul, MN, U.S.A.

Bonos, S. A., Rutgers University/Cook College, New Brunswick, NJ, U.S.A

Boudon-Padieu, E., INRA, Dijon, FRANCE

Bové, J. M., University of Bordeaux 2 La, Brede, FRANCE

Boyette, C. D., USDA ARS, Stoneville, Stoneville, MS, U.S.A.

Brannen, P. M., University of Georgia, Athens, GA, U.S.A.

Braun, U., Martin-Luther-University, Halle (Saale), GERMANY

Brown, J. K., University of Arizona, Tucson, AZ, U.S.A.

Brown, J. K. M., John Innes Centre, Norwich, UNITED KINGDOM

Burr, T., Cornell University-NYSAES, Geneva, NY, U.S.A.

Byrne, P., Colorado State University, Fort Collins, CO, U.S.A.

Cadle-Davidson, L., USDA-ARS, GGRU, Geneva, NY, U.S.A.

Calvert, L. A., CIAT, Cali, COLOMBIA

Carisse, O., Agriculture and AgriFood Canada, St-Jean-sur-Richelieu, QC, CANADA

Carson, M., USDA-ARS, St. Paul, MN, U.S.A.

Castagnone-Sereno, P., INRA, Sophia Antipolis, FRANCE

Chacko, N., University of Georgia, Athens, GA, U.S.A.

Chakraborty, S., CSIRO Brisbane, QLD, AUSTRALIA

Chang, C.-J., University of Georgia, Griffin, GA, U.S.A.

Chastagner, G. A., Washington State University, Puyallup, WA, U.S.A.

Chellemi, D. O., USDA-ARS, Fort Pierce, FL, U.S.A.

Chen, X., USDA-ARS and Washington State University, Pullman, WA, U.S.A.

Chen, J., USDA-ARS, Parlier, CA, U.S.A.

Chen, Z.-Y., Louisiana State University Agricultural Center, Baton Rouge, LA, U.S.A.

Chen, M. S., Kansas State University, Manhattan, KS, U.S.A.

Chen, S., University of Minnesota, Waseca, MN, U.S.A

Chilvers, M. I., Washington State University, Pullman, WA, U.S.A.

Ciuffetti, L. M., Oregon State University, Corvallis, OR, U.S.A.

Cohen, Y. R., Bar-Ilan University, Ramat-Gan, ISRAEL

Colditz, F., University of Bielefeld, Bielefeld, GERMANY

Cook, A., UNITED KINGDOM

Cooke, D. E. L., Scottish Crop Research Institute, Dundee, UNITED KINGDOM

Coplin, D., The Ohio State University, Columbus, OH, U.S.A.

Correa, F., RiceTec Inc., Alvin, TX, U.S.A.

Correll, J. C., University of Arkansas, Fayetteville, AR, U.S.A.
Cotty, P., USDA-ARS, Tucson, AZ, U.S.A.

Couch, B., University of Toronto, Mississauga, ON, CANADA

Coutinho, T., University of Pretoria, Pretoria, SOUTH AFRICA

Coyne, C., USDA-ARS, Pullman, WA, U.S.A.

Craemer, C., ARC-Plant Protection Research Institute, Pretoria, SOUTH AFRICA

Creamer, R., New Mexico State University, Las Cruces, NM, U.S.A.

Cubero, J., INIA, Madrid, SPAIN

Cuppels, D., Agriculture and Agri-Food Canada, London, ON, CANADA

Damsteegt, V. D., Foreign Disease-Weed Science Research Unit, Fort Detrick, MD, U.S.A.

Dasanayaka, N., University of Sri Jayewardenepura, Sri Lanka, Colombo, SRI LANKA

de Kievit, T., University of Manitoba, Winnipeg, MB, CANADA

de Wispelaere, M., University of California, Riverside, CA, U.S.A.

DeMarsay, A., University of Maryland, Upper Marlboro, MD, U.S.A.

Deom, M., University of Georgia, Athens, GA, U.S.A.

Dillard, H., NYSAES, Geneva, New York, U.S.A.

Ding, B., Ohio State University, Columbus, OH, U.S.A.

Donofrio, N., University of Delaware, Newark, DE, U.S.A.

Dorner, J., USDA-ARS, Dawson, GA, U.S.A.

Dorrance, A. E., The Ohio State University, Wooster, OH, U.S.A.

Douhan, G. W., University of California, Riverside, CA, U.S.A.

du Toit, L. J., Washington State University, Mount Vernon, WA, U.S.A.

Duncan, R. W., University of California, Davis, CA, U.S.A.

Dunkle, L., USDA-ARS/Purdue University, West Lafayette, IN, U.S.A.

Duveiller, E., CIMMYT, MEXICO

Dyer, A. T., Montana State University, Bozeman, MT, U.S.A

Dzahini-Obiatey, H. K., University of Reading, Reading, UNITED KINGDOM

Ekramoddoullah, A., Pacific Forestry Centre, Victoria, BC, CANADA

Ellis, J., CSIRO Plant Industry, Acton, AUSTRALIA

Elmer, W. H., Connecticut Agr. Exp. Station, New Haven, CT, U.S.A.

Enjalbert, J., INRA, Thiverval-Grignon, FRANCE

Esker, P., University of Wisconsin, Madison, WI, U.S.A.

Eujayl, I. A., USDA-ARS, Kimberly, ID, U.S.A.

Falk, B., University of California, Davis, CA, U.S.A.

Faris, J., Northern Crop Science Laboratory, Fargo, ND, U.S.A.

Favaron, F., University of Padova, Legnaro, ITALY

Fereres Castiel, A. Instituto Ciencias Agrarias-CSIC, Madrid, SPAIN

Fermaud, M., INRA, Villenave d'Ornon, FRANCE

Fernández-Pavía, S. P., Instituto de Investigaciones Agropecuarias y Forestales, Morelia, MEXICO

Ferris, H., University of California, Davis, CA, U.S.A.

Fetch, T., Agriculture \& Agri-Food Canada, Winnipeg, MB, CANADA

Fidelibu, M., University of California, Davis, Parlier, CA, U.S.A.

Filichkin, S., Oregon State University, Corvallis, OR, U.S.A.

Fitt, B. D. L., Rothamsted Research, Harpenden, Hertfordshire, UNITED KINGDOM

Fletcher, J., Oklahoma State University, Stillwater, OK, U.S.A.

Flint-Garcia, S., USDA-ARS, Columbia, MO, U.S.A

Forbes, G., International Potato Center, Lima, PERU

Fournier, E., INRA, Campusz International de Baillarguet, Montpellier, FRANCE

Fravel, D., USDA-ARS, BARC-West, Beltsville, MD, U.S.A.

Frederick, R., USDA-ARS FDWSRU, Fort Detrick, MD, U.S.A.

Fricke, W., University College Dublin, IRELAND

Friesen, T., Northern Crop Science Laboratory, Fargo, ND, U.S.A.

Fry, W., Cornell University, Ithaca, NY, U.S.A.

Gadoury, D., Cornell University, NYSAES, Geneva, NY, U.S.A.

Gale, L. R., University of Minnesota, St. Paul, MN, U.S.A.

Galiana, E., INRA, Antibes Cedex, FRANCE

Garbelotto, M., University of California, Berkeley, CA, U.S.A.

Garcia, R., University of Arizona, Tucson, AZ, U.S.A.

Garcia-Arenal, F., Universidad Politecnica de Madrid, Madrid, SPAIN

Gaulin, E., Universite Toulouse, Castanet-Tolosan, FRANCE

Gent, D. H., USDA-ARS, Corvallis, CO, U.S.A.

German, T., University of Wisconsin, Madison, WI, U.S.A.

Gibson, D., Cornell University, Ithaca, NY, U.S.A.

Gijzen, M., Agriculture and Agri-Food Canada, London, ON, CANADA

Gitaitis, R., University of Georgia, Tifton, GA, U.S.A.

Glenn, A. E., USDA-ARS, Athens, GA, U.S.A.

Gold, S. E., University of Georgia, Athens, GA, U.S.A. 
Golembiewski, R., University of Minnesota, Crookston, MN, U.S.A. Gonzalez, C. F., Texas A\&M University, College Station, TX, U.S.A. Gonzalez-Jaen, M. T., University Complutense of Madrid, SPAIN

Goodin, M., University of Kentucky, Lexington, KY, U.S.A.

Goss, E., USDA-ARS, West Lafayette, IN, U.S.A.

Goswami, R., North Dakota State University, Fargo, ND, U.S.A.

Goyeau, H., INRA, Thiverval-Grignon, FRANCE

Graham, J., University of Florida, Lake Alfred, FL, U.S.A.

Graham, J. H., Citrus Research and Education Center, Lake Alfred, FL, U.S.A.

Genier, E., INRA, Le Rheu, FRANCE

Grube, R. C., University of New Hampshire Coop. Ext., Durham, NH, U.S.A.

Gunasekera, T., Miami University, Oxford, OH, U.S.A.

Hammerschmidt, R., Michigan State University, East Lansing, MI, U.S.A

Hansen, E., Oregon State University, Corvallis, OR, U.S.A.

Harrison, N., University of Florida, Fort Lauderdale, FL, U.S.A.

Hartman, G., USDA-ARS and University of Illinois, Urbana, IL, U.S.A.

Hartung, J. S., USDA-ARS MPPL, Beltsville, MD, U.S.A.

Hatcher, P., Reading, UNITED KINGDOM

He, Z., Institute of Plant Physiology and Ecology, Shanghai, CHINA

Hehl, R., Technische Universität Braunschweig, Brausnchweig, GERMANY

Hiebert, E., University of Florida, Gainesville, FL, U.S.A.

Hintz, W., University of Victoria, Victoria, BC, CANADA

Hoffmeister, D., University of Minnesota, St. Paul, MN, U.S.A.

Hogenhout, S., John Innes Centre, Norwich, UNITED KINGDOM

Hollomon, D., University of Bristol, Bristol, UNITED KINGDOM

Hopkins, D., University of Florida, Apopka, FL, U.S.A.

Hua, S. S., USDA-ARS, Albany, CA, U.S.A.

Hughes, K., John Dudfield, Central Science Laboratory, York, North Yorkshire, UNITED KINGDOM

Hull, R., John Innes Centre, Norwich, UNITED KINGDOM

Hyde, K. D., Mae Fah Luang University, Chaing Rai, THAILAND

Iacobellis, N., Universita Degli Studi Della Basilicata, Potenza, ITALY

Imin, N., The Australian National University, Acton, Canberra, ACT, AUSTRALIA

Isard, S., Pennsylvania State University, University Park, PA, U.S.A.

Ishii, H., National Institute for Agro-Environmental Sciences, Tsukuba, JAPAN

Ito, Y., National Food Research Institute, Ibaraki, JAPAN

Ivors, K., North Carolina State University, Fletcher, NC, U.S.A.

Jack, A., Cornell University, Ithaca, NY, U.S.A.

Janisiewicz, W. J., USDA-Appalachian Fruit Research Station, Kearneysville, WV, U.S.A.

Jiménez-Díaz, R. M., Instituto Agricultura Sostenible, Córdoba, SPAIN

Jimenez-Gasco, M. D. M., The Pennsylvania State University, University Park, PA, U.S.A.

Jin, Y., USDA ARS, University of Minnesota, St. Paul, MN, U.S.A.

Jin, H., University of California, Riverside, CA, U.S.A.

Johnson, D. A., Washington State University, Pullman, WA, U.S.A.

Johnson, K., Oregon State University, Corvallis, OR, U.S.A.

Johnson, S. N., SCRI, Dundee, UNITED KINGDOM

Johnston, P., Landcare Research, Auckland, NEW ZEALAND

Jordan, R., US National Arboretum, USDA ARS, Beltsville, MD, U.S.A

Kacheroo, P., University of Kentucky, Lexington, KY, U.S.A.

Kaliff, M., SLU, Uppsala, SWEDEN

Kany, K., Rothamsted Research. Harpenden, UNITED KINGDOM

Karaoglanidis, G., Hellenic Sugar Industry, Platy Imathias, GREECE

Kassemeyer, H.-H., Staatliches Weinbauinstitut, Freiburg im Breisgau, GERMANY

Keinath, A., Clemson University, Charleston, SC, U.S.A.

Keller, B., University of Zurich, Zürich, SWITZERLAND

Kenerley, C., Texas A\&M University, College Station, TX, U.S.A

Kennedy, R., Warwick University, Warwick, Warwickshire, UNITED KINGDOM

Kennedy, G. G., North Carolina State University, Raleigh, NC, U.S.A.

Kerry, B. R., Rothamsted Research, Harpenden, UNITED KINGDOM

Kessel, G., Plant Research International, Wageningen, NETHERLANDS

Kirk, W., Michigan State University, East Lansing, MI, U.S.A.

Kirkpatrick, B., University of California, Davis, CA, U.S.A.

Kleckowski, A., University of Stirling, Stirling, UNITED KINGDOM

Kliebenstein, D., University of California, Davis, CA, U.S.A.

Kloepper, J., Auburn University, Auburn, AL, U.S.A.

Ko, W.-H., National Chung Hsing University, Taichung, TAIWAN, PROVINCE OF CHINA

Kobayashi, N., IRRI, Los Banos, Laguna, PHILIPPINES

KODETHAM, G., University of Hyderabad, Hyderabad, Andhra Pradesh, INDIA

Köhl, J., Plant Research International, Wageningen, NETHERLANDS

Kohn, L., University of Toronto, Mississauga, ON, CANADA

Kolmer, J., USDA-ARS, University of Minnesota, St. Paul, MN, U.S.A.

Korolev, N., ARO-Volcani Center, Bet Dagan, ISRAEL

Kox, L. F. F., Plant Protection Service, Wageningen, NETHERLANDS

Lakshman, D., USDA-ARS, Beltsville, MD, U.S.A.

Lamari, L., University of Manitoba, Winnipeg, MB, CANADA

Lapidot, M., Volcani Center, ARO, Bet Dagan, ISRAEL
Latorre, B., Pontificia Universidad Catolica de Chile, Casilla, Santiago, CHILE

Lee, R. F., USD-ARS, Riverside, CA, U.S.A.

Lees, A., SCRI, Dundee, UNITED KINGDOM

Lefort, F., University of Applied Sciences of Western Switzerland, Jussy, Geneva, SWITZERLAND

Lehmensiek, A., Queensland Department of Primary Industry and Fisheries, Toowoomba, QLD, AUSTRALIA

Lei, Z., The Samuel Roberts Noble Foundation, Ardmore, OK, U.S.A.

Li, B., Dupont Company

Lievens, B., Scientia Terrae Research Institute, Sint-Katelijne-Waver, BELGIUM

Lilley, C., University of Leeds, Leeds, West Yorkshire, UNITED KINGDOM

Linderman, R., Plant Health LLC, Corvallis, OR, U.S.A.

Lindow, S., University California, Berkeley, CA, U.S.A.

Liu, Z., USDA-ARS, Fargo, ND, U.S.A.

López, M., Milagros, I.V.I.A, Moncada, Valencia, SPAIN

Lori, C., Washington State University, Pullman, WA, U.S.A

Lorito, M., Università degli Studi di Napoli Federico II, Portici, ITALY

Lulai, E., USDA ARS, Northern Crop Science Lab., Fargo, ND, U.S.A.

Luo, Y., University of California, Kearney Agricultural Center, Parlier, CA, U.S.A.

Luster, D. G., USDA-ARS, Fort Detrick, MD, U.S.A.

Luthe, D., Penn. State University, University Park, PA, U.S.A.

Ma, Z., Hangzhou, CHINA

Madden, L. V., Ohio State University, Wooster, OH, U.S.A.

Maixner, M., Biologische Bundesanstalt, Bernkastel-Kues, GERMANY

Malvick, D., University of Minnesota, St. Paul, MN, U.S.A.

Manicom, B., ARC-ITSC, Nelspruit, SOUTH AFRICA

Manjunath, K. L., USDA, Riverside, CA, U.S.A.

Marçais, B., INRA, Champenoux, FRANCE

Marcos, J. F., IATA-CSIC, Burjassot, Valencia, SPAIN

Marois, J., University of Florida, Quincy, FL, U.S.A.

Matheron, M. E., University of Arizona, Yuma, AZ, U.S.A.

Mathews, D., University of California, Riverside, CA, U.S.A.

Matthews, B., U.S. Department of Agriculture, Beltsville, MD, U.S.A

Mazzola, M., USDA-ARS, Wenatchee, WA, U.S.A.

McCallum, B., Agriculture and Agri-Food Canada, Winnipeg, MB, CANADA

MacDonald, R. M., University of Guelph, CANADA

McSpadden G. B., Ohio State University-OARDC, Wooster, OH, U.S.A.

Melcher, U. K., Oklahoma State University, Stillwater, OK, U.S.A.

Mellon, J., USDA-ARS SRRC, New Orleans, LA, U.S.A.

Menzies, J., Agriculture and Agri-Food Canada, Winnipeg, MB, CANADA

Mergoum, M., North Dakota State University, Fargo, ND, U.S.A.

Miklas, P., USDA-ARS IAREC, Prosser, WA, U.S.A.

Mila, A., North Carolina State University, Raleigh, NC, U.S.A.

Miles, M., USDA-ARS, Champaign, IL, U.S.A.

Milgroom, M. G., Cornell University, Ithaca, NY, U.S.A

Miller, S., The Ohio State University, Wooster, OH, U.S.A.

Mitchell, C., Univ of North Carolina, Chapel Hill, NC, U.S.A.

Mou, Z., University of Florida, FL, U.S.A.

Mueller, D., Iowa State University, Ames, IA, U.S.A.

Mundt, C., Oregon State University, Corvallis, OR, U.S.A.

Munkvold, G., Iowa State University, Ames, IA, U.S.A.

Murillo, J., Universidad Publica de Navarra, Pamplona, SPAIN

Navazio, J., Prescott College, Prescott, AZ, U.S.A.

$\mathrm{Ng}$, J., University if California, Riverside, CA, U.S.A.

Ngugi, H., The University of Georgia, Athens, GA, U.S.A.

Norelli, J. L., USDA-ARS, Kearneysville, WV, U.S.A.

Nunney, L., University of California, Riverside, CA, U.S.A.

O'Donnell, K., NCAUR-USDA ARS, PEORIA, IL, U.S.A.

Ohm, H., Purdue University, West Lafayette, IN, U.S.A.

Okubara, P. A., USDA-ARS, Washington State University, Pullman, WA, U.S.A.

Owens, R., Beltsville Agricultural Research Center, Beltsville, MD, U.S.A.

Palm-Hernandez, M. E., USDA, Beltsville, MD, U.S.A.

Panaccione, D., West Virginia University, Morgantown, WV, U.S.A.

Pappu, H., Washington State University, Pullman, WA, U.S.A.

Parlevliet, J., Laboratory of Plant Breeding, Wageningen, NETHERLANDS

Pastor-Corrales, M. A., USDA-ARS, Beltsville, MD, U.S.A.

Pataky, J., University of Illinois, Urbana, IL, U.S.A.

Paul, P. A., Ohio State University, Wooster, OH, U.S.A.

Paulitz, T. C., USDA-ARS, Washington State University, Pullman, WA, U.S.A.

Payne, G., North Carolina State University, Raleigh, NC, U.S.A.

Peever, T., Washington State University, Pullman, WA, U.S.A.

Perez-Sierra, A., University Politecn Valencia, Valencia, SPAIN

Perry, K. L., Cornell University, Ithaca, NY, U.S.A.

Pertot, I., Istituto Agr. Di San Michele All'adige, S Michele A/Adige, ITALY

Pfender, W., USDA NFSPRC, Corvallis, OR, U.S.A.

Pierret, A., IRD-IWMI-NAFRI, Vientiane, Lao People's Democratic Republic

Pierson, L. S., University of Arizona, Tucson, AZ, U.S.A.

Pinkerton, J., USDA-ARS, Corvallis, OR, U.S.A.

Pinnschmidt, H., Danish Inst. Agric. Science, Slagelse, DENMARK

Ploetz, R. C., University of Florida, Homestead, FL, U.S.A.

Powers, T. O., University of Nebraska, Lincoln, NE, U.S.A. 
Price, A., University of Aberdeen, Aberdeen, UNITED KINGDOM Proctor, R., USDA-ARS NCAUR, Peoria, IL, U.S.A. Prospero, S., Swiss Federal Research Inst. WSL, Birmensdorf, SWITZERLAND Prusky, D., The Volcani Center, Rishon Le Tzion, ISRAEL

Pryor, B., University of Arizona, Tucson, AZ, U.S.A.

Putnam, M., Oregon State University, Corvallis, OR, U.S.A

Raaijmakers, J., Wageningen University, Wageningen, NETHERLANDS

Raccah, B., The Volcani Center, Bet Dagan, Rishon Lezion, ISRAEL

Raghu, S., Queensland University of Technology, Brisbane, QLD, AUSTRALIA

Raman, H., NSW Department of Primary Industry, AUSTRALIA

Rashid, K. Y., Agricultural and Agri-Food Canada, Morden, MB, CANADA

Rayapati, N., Washington State University, Prosser, WA, U.S.A.

Restrepo, S., Universidad de los Andes, Bogotá, COLOMBIA

Ristaino, J. B., North Carolina State University, Raleigh, NC, U.S.A.

Ritchie, D., North Carolina State University, Raleigh, NC, U.S.A.

Rizzo, D. M., University of California, Davis, CA, U.S.A.

Roberts, D. P., USDA-ARS, Beltsville, MD, U.S.A

Roberts, P. A., University of California, Riverside, CA, U.S.A.

Robertson, D., North Carolina State University, Raleigh, NC, U.S.A.

Rollins, J. A., University of Florida, Gainesville, FL, U.S.A.

Roncero, M. I. G., University of Cordoba, Cordoba, SPAIN

Roper, C., University of Connecticut, Connecticut, RI, U.S.A.

Rowe, R. C., Ohio State University, Wooster, OH, U.S.A.

Ruiz-Medrano, R., CINVESTAV-IPN, Colonia San Pedro Zacatenco, Mexico DF, MEXICO

Rupe, J. C., University of Arkansas, Fayetteville, AR, U.S.A.

Sache, I., INRA, Thiverval-Grignon, FRANCE

Sagaram, U. S., Texas A\&M University, College Station, TX, U.S.A.

Samuels, G., USDA-ARS, Beltsville, MD, U.S.A.

Savary, S., IRRI, Metro Manila, Los Banos, Laguna, PHILIPPINES

Schaad, N., USDA-ARS, Fort Detrick, MD, U.S.A.

Schart, J., Schlossgarten 3 Münster, GERMANY

Scherm, H., University of Georgia, Athens, GA, U.S.A.

Schmale, D., Virginia Polytechnic Institute and State University, Blacksburg, VA, U.S.A.

Schnabel, G., Clemson University, Clemson, SC, U.S.A.

Schneider, R. W., Louisiana State University, Baton Rouge, LA, U.S.A.

Schneider, W., USDA, Fort Detrick, MD, U.S.A

Schnell, R. J., USDA-ARS, Miami, FL, U.S.A.

Scholthof, H., Texas A\&M University, College Station, TX, U.S.A.

Schottel, J., University of Minnesota, St. Paul, MN, U.S.A.

Seebold, K. W., University of Kentucky, Lexington, KY, U.S.A.

Seemueller, E., Biologische Bundesanstalt, Dossenheim, GERMANY

Shah, D. A., Lewiston, NY, U.S.A.

Shamoun, S. F., Canadian Forest Service, Victoria, BC, Victoria, BC, CANADA

Shaner, G., Purdue University, West Lafayette, IN, U.S.A.

Sharon, A., Tel Aviv University, Tel Aviv, ISRAEL

Shaw, M., University of Reading, Reading, UNITED KINGDOM

Shaw, M. New Mexico Highlands University, Las Vegas, NM, U.S.A.

Shim, W.-B., Texas A\&M University, College Station, TX, U.S.A.

Shishkoff, Nina, USDA-ARS FDWSRU, Frederick, MS, U.S.A.

Shtienberg, D., ARO, The Vocani Center, Bet Dagan, ISRAEL

Singh, P. K., North Dakota State University, Fargo, ND, U.S.A.

Singh, K. P., Raja Balwant Singh, Agra, INDIA

Singh, V., IISR,Lucknow-INDIA, Lucknow, INDIA

Sivasithamparam, K., The University of Western Australia, Perth, WA, AUSTRALIA

Skantar, A., USDA-ARS, Beltsville, MD, U.S.A.

Smart, C. D., Cornell University, Geneva, NY, U.S.A.

Smith, S. M., University of Georgia, Athens, GA, U.S.A.

Solfrizzo, M., National Research Council, Bari, ITALY

Spirodonov, S. E., Institute of Parasitology, RAS, Moscow, RUSSIA

Spoel, S., Duke University, Durham, NC, U.S.A.

Stammler, G., BASF-AG, Limburgerhof, GERMANY

Stanghellini, M., University of California, Riverside, CA, U.S.A.

Starr, J. L., Texas A\&M University, College Station, TX, U.S.A.

Steffenson, B. J., University of Minnesota, St. Paul, MN, U.S.A.

Stenger, D. C., USDA-ARS San Joaquin Valley Agricultural Sciences Center,

Parlier, CA, U.S.A.

Stirling, G., Biological Crop Protection, Moggill, QLD, AUSTRALIA

Stockwell, V., Oregon State University, Corvallis, OR, U.S.A.

Stone, J. K., Oregon State University, Corvallis, OR, U.S.A.
Strausbaugh, C., USDA-ARS, Kimberly, ID, U.S.A.

Subbarao, K. V., University of California, Davis, Salinas, CA, U.S.A.

Sugar, D., Oregon State University, Medford, OR, U.S.A.

Sullivan, R., Rutgers University, New Brunswick, NJ, U.S.A.

Summerell, B. A., Royal Botanic Gardens and Domain Trust, Sydney, NSW, AUSTRALIA

Sundin, G. W., Michigan State University, East Lansing, MI, U.S.A.

Sutton, T. B., North Carolina State University, Raleigh, NC, U.S.A.

Suty, L., INRA, Dijon, FRANCE

Suzuki, N., Okayama University, Kurashiki, JAPAN

Szabo, L. J., USDA-ARS, St. Paul, MN, U.S.A

Talhinhas, P., Instituto Superior de Agronomia, Lisboa, PORTUGAL

Tatineni, S. C., University of Florida, Lake Alfred, FL, U.S.A.

Tebbs, J., University of South Carolina, Columbia, SC, U.S.A.

Templeton, M., Hort Research, Sandringham, Auckland, NEW ZEALAND

Thomashow, L., USDA-ARS, Pullman, WA, U.S.A.

Thwaites, R., Natural Resources Inst., Chatham, Kent, UNITED KINGDOM

Timmer, P., University of Florida, Lake Alfred, FL, U.S.A.

Tooley, P., USDA-ARS FDWSRU, Fort Detrick, MD, U.S.A.

Tosa, Y., Kobe University, Kobe, Hyogo, JAPAN

Tredway, L. P., North Carolina State University, Raleigh, NC, U.S.A.

Tsopelas, P., Institute of Mediterranean Forest Ecosystems, Athens, GREECE

Turechek, W. W., USDA-ARS USHRL, Fort Pierce, FL, U.S.A.

Tzanetakis, I., University of Arkansas, Fayetteville, AR, U.S.A.

Tzortzakakis, E. A., Plant Protection Institute, Heraklion, Crete, GREECE

Unruh, T., USDA-ARS, Wapato, WA, U.S.A.

Urwin, P., University of Leeds, Leeds, UNITED KINGDOM

Valkonen, J., University of Helsinki, Helsinki, FINLAND

van den Bosch, F., Rothamsted Research, Harpenden, UNITED KINGDOM

van der Linden, G., Plant Research International, Wageningen, NETHERLANDS

Vandemark, G., USDA-ARS, Pullman, WA, U.S.A.

Vasquez, S., University of California, Fresno, CA, U.S.A.

Vaz Patto, M. C., Instituto de Tecnologia Química e Biológica, ITQB, Oeiras, PORTUGAL

Venette, R., USDA Forest Service, St. Paul, MN, U.S.A.

Verchot-Lubicz, J., Oklahoma State University, Stillwater, OK, U.S.A.

Viret, O., ACW-Changins, Nyon, SWITZERLAND

Vettraino, A. M., Università degli Studi della Tuscia, Viterbo, ITALY

Voegele, R. T., University of Konstanz, Konstanz, BW, GERMANY

von Tiedemann, A., University of Gottingen, Goettingen, GERMANY

Wallwork, H., South Australian Research and Development Institute, Adelaide, AUSTRALIA

Wang, G., Ohio State University, Columbus, OH, U.S.A.

Wang, N., University of Florida, Lake Alfred, FL, U.S.A.

Wanner, L. A., USDA-Agricultural Research Service, Beltsville, MD, U.S.A.

Ward, T., USDA-ARS, Peoria, IL, U.S.A.

Wegulo, S., University of Nebraska, Lincoln, NE, U.S.A.

Weiland, J., USDA-ARS, Corvallis, OR, U.S.A.

Wellings, C., University of Sydney, Camden, NSW, AUSTRALIA

Wen, A., University of Florida, Quincy, FL, U.S.A.

Whipps, J. M., University of Warwick, Warwick, UNITED KINGDOM

White, F., Kansas State University, Manhattan, KS, U.S.A.

Wilson, M., Colorado College, Colorado Springs, CO, U.S.A

Windes, J. M, University of Idaho, Idaho Falls, ID, U.S.A.

Wingfield, B., University of Pretoria, Pretoria, SOUTH AFRICA

Wisniewski, M., USDA-ARS, Kearneysville, WV, U.S.A.

Wolpert, T., Oregon State University, Corvallis, OR, U.S.A.

Wolyn, D., University of Guelph, Guelph, ON, CANADA

Woodward, S., University of Aberdeen, Aberdeen, UNITED KINGDOM

Wu, W., Zhejiang University, Hangzhou, Zhejiang, CHINA

Wydra, K., Institute of Plant Diseases and Plant Protection, Hannover, GERMANY

Yacaman, M., University of Texas, San Antonio, TX, U.S.A.

Yang, X. Southwest University, Beibei, Chongqing, CHINA

Yasui, H., Kyushu University, Fukuoka, JAPAN

Yuen, J. E., Swedish University of Agric Science, Uppsala, SWEDEN

Yun, D.-J., Gyeongsang National University, Jinju, REPUBLIC OF KOREA

Zeller, K., USDA-APHIS-PPQ-CPHST, Beltsville, MD, U.S.A.

Zerbini, M., Universidade Federal de Vicosa, Campus Universitario, Vicosa, Minas Gerais, BRAZIL

Zeyen, R. J., University of Minnesota, St. Paul, MN, U.S.A

Zhang, S., University of Kentucky, Lexington, KY, U.S.A.

Zhang, D., USDA-Beltsville, Beltsville, MD, U.S.A. 